\title{
THE USE OF IMTEC'S MDI MINI-IMPLANTS IN THE TREATMENT OF TOTALLY EDENTULOUS MANDIBLE
}

\author{
Metodi Abadzhiev, Miglena Balcheva \\ Prosthodontic Department, Faculty of Dental Medicine, \\ Medical University - Varna
}

\section{SUMMARY:}

It is a hard task to produce full mandibular dentures for highly atrophic alveolar ridges. The lack of alveolar bone and attached gingiva enormously reduces the functional suitability of the dentures. The implantologic treatment is an often ignored opportunity, because of the necessity of additional surgical intervention for ridge augmentation and the advanced age of the patients. Thus implants with diameter $1.8-2.2 \mathrm{~mm}$ with spherical attachments for removable over-implant denture retention are possible alternative for the patients. denture

Key words: MDI, attached gingiva, over-implant

\section{PURPOSE:}

In the study are presented clinical cases with miniimplants with spherical joints for retention of removable overimplant mandibular dentures.

\section{METHODS AND MATERIALS:}

Patients at average age of 72. MDI implants with diameter $2.1 \mathrm{~mm}$, length 10.0 or $13.0 \mathrm{~mm}$ and spherical attashment, placed flapless in the mandible in the mentum region. (Fig. 1.) The implants are loaded immediately with full over-implant dentures. The dentures are made on IVOCAP technology in advance.

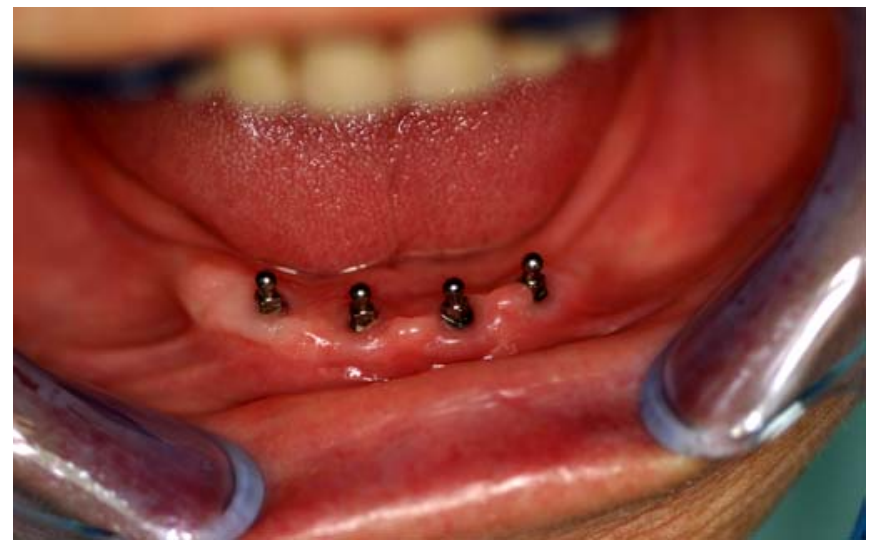

Fig. 1. MDI after the placement)

\section{RESULTS:}

There is no swelling or pain in the postoperative period. The patients feel comfortable, because of the immediate denture placement and the chance to eat right after the surgery. Three years later there is no clinical or X-ray evidence about bone resorption. The retention rings are changed every 12 months.

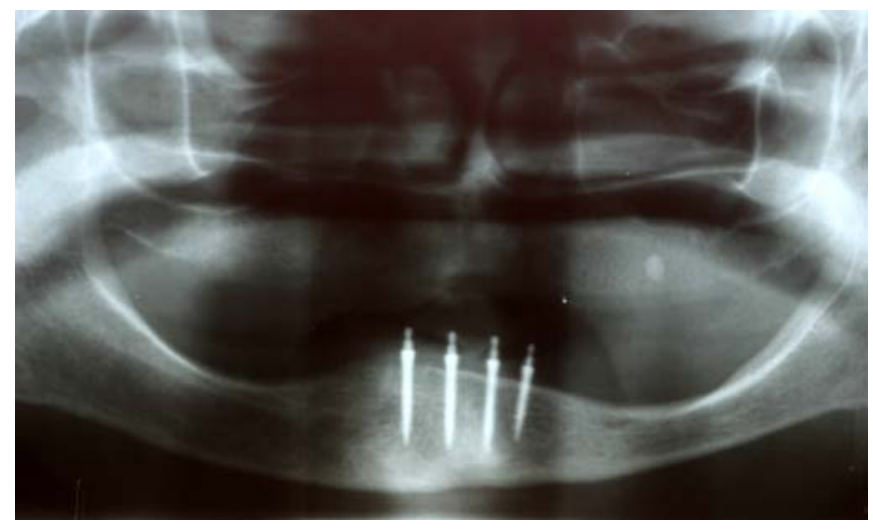

Fig. 2. OPG 2 years after the placement of the MDI

\section{DISCUSSION:}

This approach is particularly suitable for elderly patients or for ones with serious general disorders. It is a way to avoid highly invasive surgical interventions. The surgical and prosthetic protocol is easy to perform, even for general dental practitioners. It is important to pay attention to the necessity of high initial stability of the implant. On the other hand, the insufficient instrumentation of the osteotome opening could lead to implant's breaking (due to its small diameter).

Lingual piercing of the denture is possible at the matrtices' fixation. It's not a big problem - the hole is filled with self-curing resin. (Fig. 3.) This however weakens the mechanical strength of the denture and it could break. It is also possible the self-curing resin to penetrate sublingually at the fixation.That's way the implants should be covered with foil while the resin curing. 


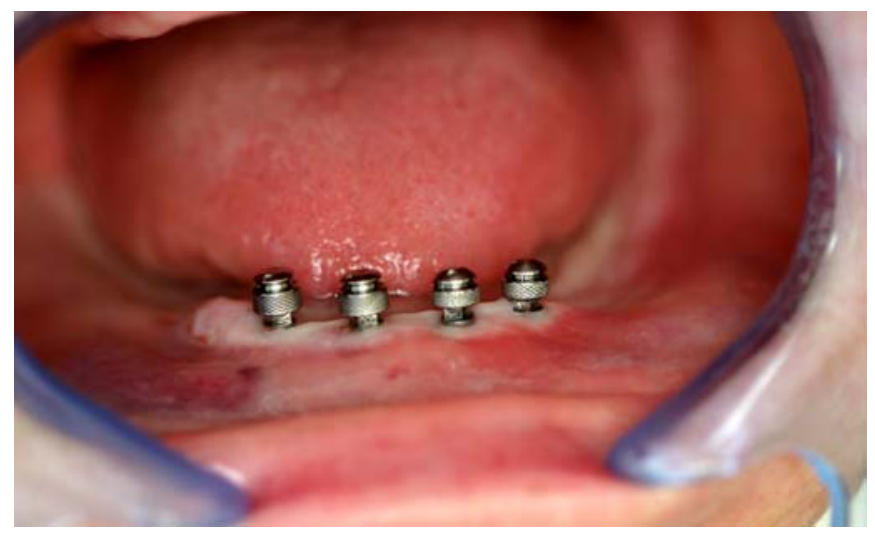

Fig. 3. The matrices before fixation

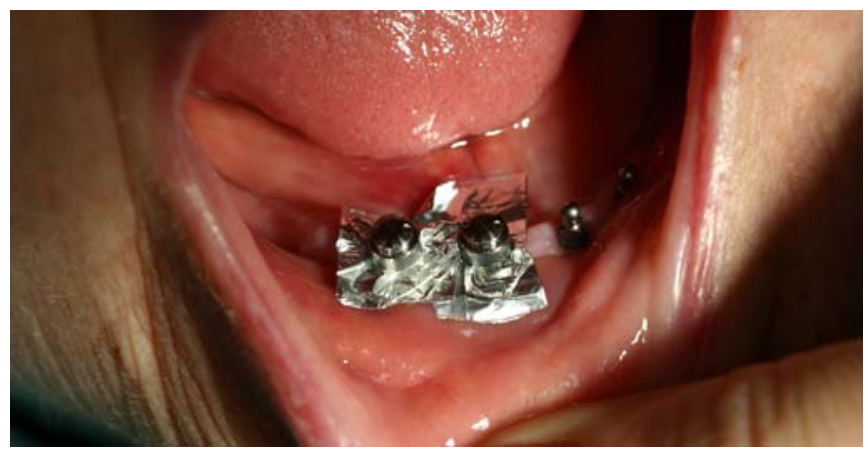

Fig. 4. The aluminium foil protects the sulcus from resin's penetration.

Fig. 6.

OPG of the most difficult for treating with full prosthesis clinical case - totally edentulous mandible with advanced resorption and maxilla with natural dentition by 76 years old patient. MDI with spherical atashment cud be a great possibility for retention of the dentures.

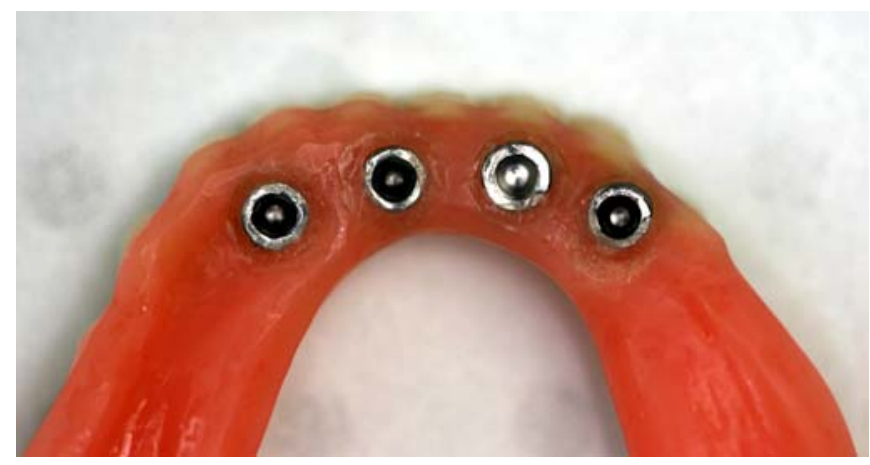

Fig. 5. The matrices, fixed in the denture

\section{CONCLUSIONS:}

The use of mini-implants with spherical joints for full mandibular dentures retention is a routine,time-proven method. It is recommended for elderly patients with marked atrophy of the alveolar ridge and variety of reasons (health, financial, psychological) for avoiding highly invasive surgical techniques.

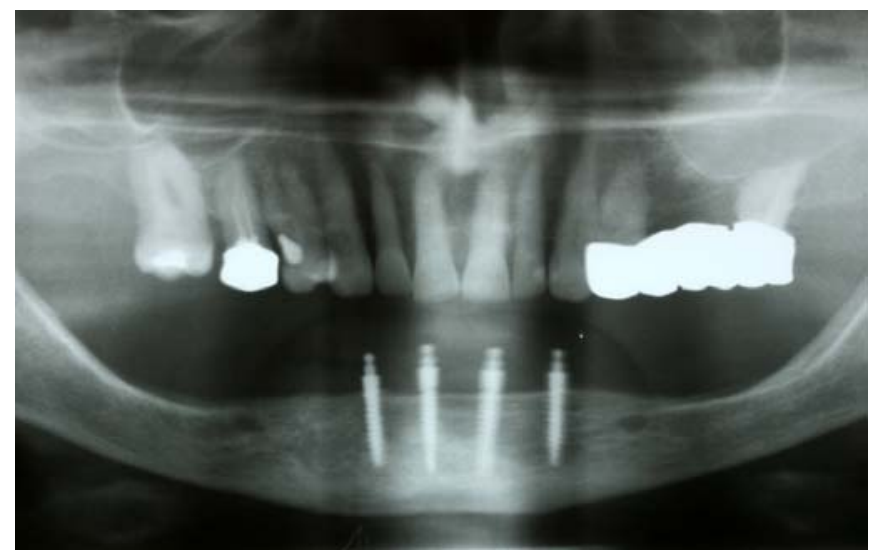

\section{REFERENCES:}

1. A 10-year randomized clinical trial on the influence of splinted and unsplinted oral implants retaining mandibular overdentures: peri-implant outcome.Naert I., Alsaadi G., van Steenberghe D., Quirynen M. Int J Oral Maxillofac Implants. 2004 Sep-Oct; 19(5) :695-702.

2. A long-term study of implants supporting overdentures as a model for implant success. Schwartz-Arad D., Kidron N., Dolev E. J Periodontol. 2005 Sep; 76(9): 1431-5
3. Immediate loading of two implants supporting a ball attachment-retained mandibular overdenture: a prospective clinical study.Marzola R., Scotti R., Fazi G., Schincaglia G. P. Clin Implant Dent Relat Res. 2007 Sep; 9(3):136-43.

4. A prospective study of immediately loaded single implant-retained mandibular overdentures: preliminary one-year results. Liddelow G. J., Henry P. J. J Prosthet Dent. 2007 Jun; 97(6 Suppl):S126-37. Erratum in:

\section{J Prosthet Dent. 2008 Mar;99(3):167.}

5. Solutions for Severely Angulated Implants in the Mandibular Overdenture: A Clinical Report. Akkad S., Richards M. J Prosthodont. 2008 Nov 18

6. Height parallelism of implants in the treatment of the edentulous mandible with ball-retained overdentures: a technical note. Iglesia-Puig M. A. Int J Oral Maxillofac Implants. 2008 Sep-Oct;23(5):953-4.

\section{Address for correspondence:}

Metodi Abadzhiev

Prosthodontic Department, Faculty of Dental Medicine, Medical University - Varna 63, G. S. Rakovski Str., 9000 Varna, Bulgaria

E-mail: mabadjieff@yahoo.com 